\title{
DETERMINING THE POSITION AND STATE OF POST-IMPACT MOTION OF A MOTOR VEHICLE STRUCK ON ITS SIDE AT A ROAD INTERSECTION, BASED ON EXPERIMENTAL TESTS
}

\author{
Mirosław GIDLEWSKI ${ }^{1,2^{\star}}$, Leon PROCHOWSKI ${ }^{3,4}$, Wojciech $\mathrm{WACH}^{5}$ \\ ${ }^{1,4}$ Automotive Industry Institute, Warsaw, Poland \\ ${ }^{2}$ Kazimierz Pułaski University of Technology and Humanities, Radom, Poland \\ ${ }^{3}$ Military University of Technology, Warsaw, Poland \\ ${ }^{5}$ Professor Jan Sehn Institute of Forensic Research, Cracow, Poland
}

Received 1 September 2016; revised 1 December 2016; accepted 16 December 2016

\begin{abstract}
The modelling, simulation, and reconstruction of road accidents are difficult processes, where experimental test results must be used at different stages of the work. Such data are needed e.g. to create or improve models of vehicle collision dynamics and to validate the results of computer simulations of vehicle motion during and after the collision. Within the work, experimental tests were carried out and the test results were utilized for determining specific values of the parameters that characterize the state of motion of the impacted vehicle during the collision and after the vehicle separation from each other. The test results make it possible to determine the initial values to be used in calculations of further free motion of the vehicle after a side impact. In particular, they enable defining the influence of the location of the point of impact against the vehicle side on the position and angle of rotation of the vehicle in relation to the global reference frame and on the value, direction, and sense of the vector of linear velocity of the centre of vehicle mass and the vector of angular velocity of the vehicle body around the vertical axis. These data constitute information sufficient to determine the energy of translation and rotation of the vehicle struck on its side at a road intersection and to carry out a simulation of the vehicle motion immediately following the collision.
\end{abstract}

Keywords: transport, road traffic safety, collision, vehicle, road accident, modelling, reconstruction.

\section{Notations}

$a_{x B}, a_{y B}, a_{z B}-$ components of the vector of acceleration of the centre of mass of Vehicle $B$ in the local coordinate system $O_{B} x_{B} y_{B} z_{B}$;

$k=1, \ldots, 4-$ numbers of the time intervals within which the functions $W(t)$ were averaged;

$L_{A B}$ - distance between the longitudinal plane of symmetry of Vehicle A and the front wheel axis of Vehicle B at the instant of the collision;

$n=1, \ldots, 6$ - numbers of successive crash tests;

$P_{B}, O_{B}, R_{B}$ - components of the vector of angular velocity of the body of Vehicle B in the local coordinate system;

$R^{2}$ - coefficient of determination of the regression function models; $t$ - time;

$T_{k}$ - time intervals used when calculating the average values of functions $W(t)$;

$W(t)$ - characteristic functions represented by the curves shown in Figures 4 and 6;

$W\left(T_{k}\right)$ - average value of function $W(t)$, calculated for time interval $T_{k}$;

$W_{n k}$ - average value of function $W(t)$, calculated for time interval $T_{k}$ and the $n$-th crash test;

$\left\{V_{A}\right\}$ - vector of the velocity of Vehicle $\mathrm{A}$ at the instant of the collision;

$\bar{V}_{A}$ - average value the velocity of Vehicle A, calculated for all the crash tests;

$\left\{V_{O B}\right\}$ - centre of mass velocity vector of Vehicle B during the impact;

\footnotetext{
${ }^{\star}$ Corresponding author. E-mail: miroslaw.gidlewski@uthrad.pl
} 


$$
\begin{aligned}
& \left\{V_{w}\right\}=\left\{\dot{X}_{G B}, \dot{Y}_{G B}\right\}^{T}-\text { value and components of the } \\
& X_{G B}, Y_{G B}, Z_{G B} \text { - coordinates of the centre of } \\
& \ddot{X}_{G B}, \ddot{Y}_{G B}, \ddot{Z}_{G B} \text { - components of the Vehicle B cen- } \\
& \text { tre of mass acceleration vector } \\
& \text { in the global coordinate system; } \\
& \alpha \text { - angle of deviation of the vector } \\
& \text { of velocity } V_{w} \text { from the direc- } \\
& \text { tion of pre-impact motion of } \\
& \text { Vehicle B; }
\end{aligned}
$$

\section{Introduction}

Side collisions of motor vehicles amount to more than $25 \%$ of road accidents in Poland. Although the number of such accidents declines as in other countries (Seidl, Tomek 2015), they still constitute an important problem. To acquire knowledge of the course of accidents of this kind, experimental tests were prepared and carried out and results of these tests will be used to improve the methods of modelling and reconstruction of road accidents. There are many publications with results of measurements of side collision parameters where experiment results are used for the analysis of a specific road accident (Johnson, Gabler 2011) or are compared with results of simulation calculations in specialist computer programs (Bailey et al. 2000; Brach, R. M., Brach, R. 2011; Brach et al. 2007; Cliff, Moser 2001; McHenry, B., McHenry, R. 1997).

In this study, the values of the parameters that characterize the state of motion of the impacted vehicle will be determined by an analysis of measurement and calculation results. The data obtained from the analysis will make it possible to determine the initial values to be used in calculations of further free motion of Vehicle B after a side impact (Figure 1). In particular, the calculation results will enable defining the influence of the location of the point of impact against the vehicle side on the following:

- curves showing changes in the velocity of the centre of vehicle mass, in the angle of deviation of the velocity vector from the direction of motion of Vehicle B before the collision, and in the angular velocity of the vehicle body around the vertical axis;

- lateral displacement of the centre of vehicle mass, angle of rotation of the body of Vehicle B, and trajectory of the centre of vehicle mass.
The said calculation results will unequivocally show the position and state of motion of the vehicle, depending on the location of the point of impact against the vehicle side. The relation between the quantities that characterize the location of the point of impact and the parameters of motion of the impacted vehicle will be determined from the regression function, which is based on results of experimental tests. An analysis of this issue was undertaken in research by Gidlewski et al. (2015), where the influence of the point of impact against the vehicle side on the lateral displacements of vehicle occupants has been shown.

In this work, experimental test results have been used, which included time histories of the acceleration of the centre of vehicle mass and time histories of the angular velocity of the vehicle body, measured in the local coordinate system attached to the vehicle. The measurement and calculation results have been presented for the period $0 \ldots 0.2 \mathrm{~s}$. This is the time interval after which the vehicles separate from each other and their further motion is determined by their kinetic energy. Within this period, the displacement of the centre of vehicle mass does not exceed $1.4 \mathrm{~m}$. The velocity changes and the vehicle body motion were analysed in the global coordinate system.

\section{Preparation and carrying out of experimental tests}

Six crash tests were performed at the Automotive Industry Institute (Warsaw, Poland) with the use of 12 passenger cars of the same make and model. In each test, the front of Vehicle A crashed into the left side of Vehicle B. The pre-impact speed of Vehicle A was about $50 \mathrm{~km} / \mathrm{h}$ and it was twice as high as that of Vehicle B. The crash tests were carried out on a test yard with dry concrete surface, in good weather conditions. During the tests, the steering wheels of both cars were left free and their road wheels were not braked.

The relative positions of Vehicle A and Vehicle B were changed in successive crash tests (Figure 1). The location of the point of impact on the side of Vehicle B was defined by the distance $L_{A B}$ (Figure 2, Table 1). Values of the characteristic parameters of successive crash tests have been specified in Table 1.

The tests were carried out with the use of Honda Accord cars manufactured in 2000-2002. The cars were in good technical condition and had undamaged and noncorroded bodies, which had not been previously repaired. At the centre of mass of each car, there was a three-axial acceleration sensor, installed together with sensors measuring the angular velocity of the vehicle body in relation to the three coordinate axes whose directions and senses were consistent with those of the local coordinate systems attached to the cars.

Table 1. Velocity of Vehicle A at the instant of the collision

\begin{tabular}{|l|l|l|l|l|l|l|}
\hline$L_{A B}[\mathrm{~m}]$ & 0.04 & 0.17 & 1.25 & 1.34 & 2.57 & 2.71 \\
\hline$V_{A}[\mathrm{~m} / \mathrm{s}]$ & 13.6 & 13.1 & 15.1 & 12.7 & 14.2 & 14.7 \\
\hline
\end{tabular}


a)

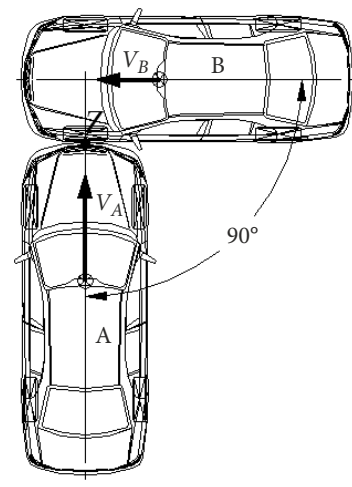

b)

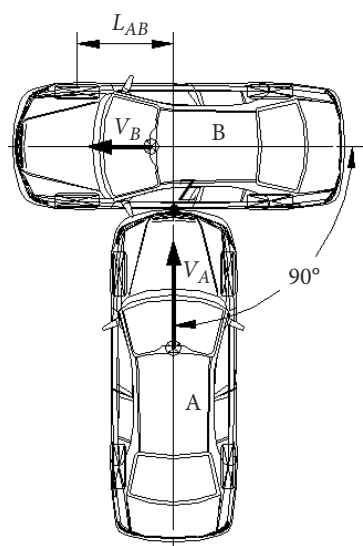

c)

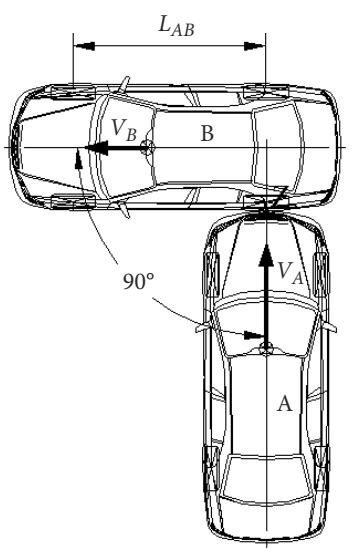

Figure 1. Relative positions of Vehicle A and Vehicle B during the crash tests

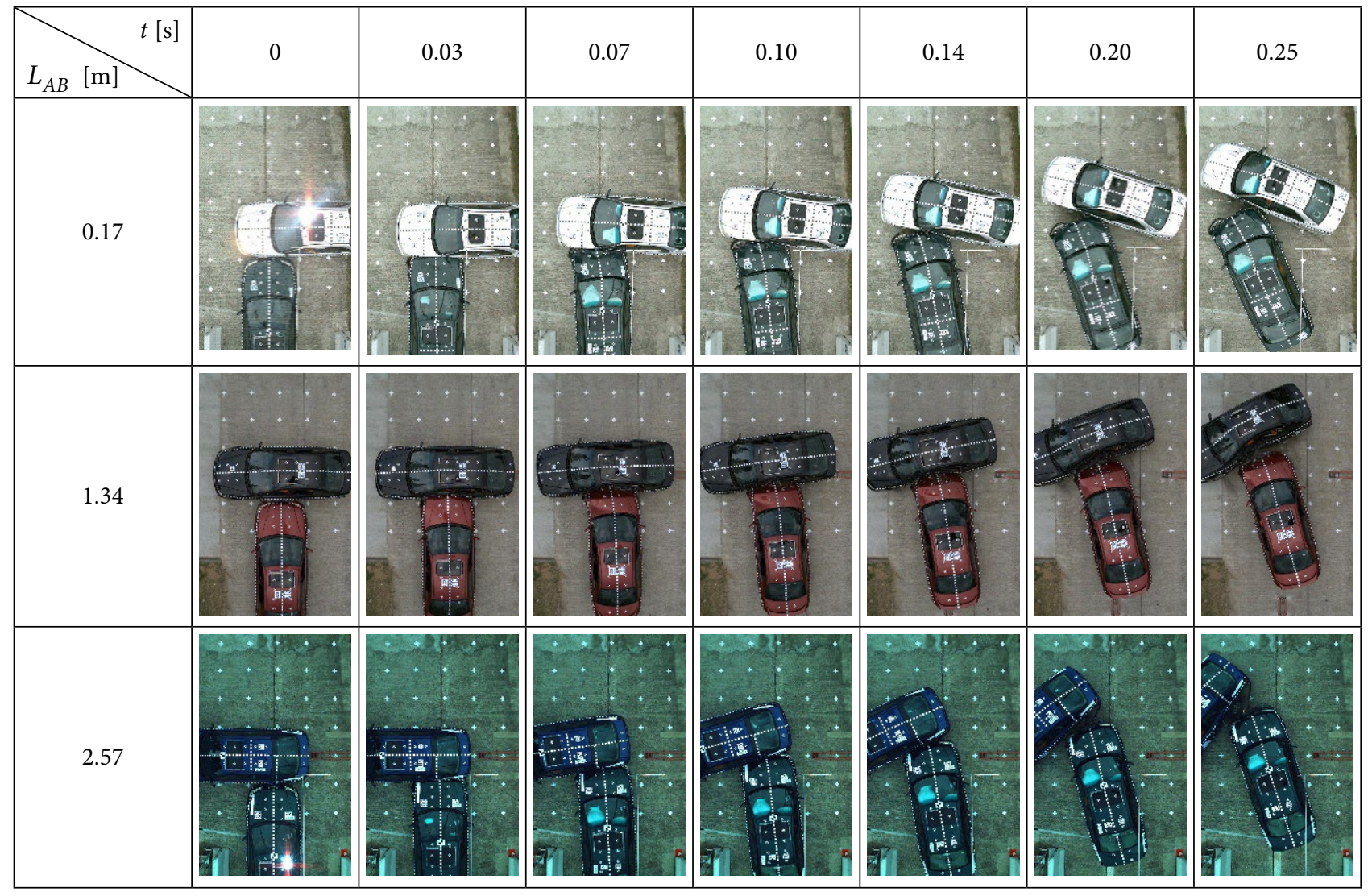

Figure 2. Positions of the cars in the initial phase of the crash tests

\section{Reference frames adopted to describe the motion of the vehicles involved}

To analyse the processes that took place during the vehicle collision, a global coordinate system and local coordinate systems were adopted. The global coordinate system $O_{G} X_{G} Y_{G} Z_{G}$ is attached to the road. The $O_{G} X_{G} Y_{G}$ plane of this system is situated at the road surface level and the $\mathrm{O}_{\mathrm{G}} \mathrm{Z}_{\mathrm{G}}$ axis is pointing vertically upwards. The $O_{G} X_{G}$ axis is parallel to the vector of pre-impact velocity of Vehicle $\mathrm{A}$ and the $O_{G} Y_{G}$ axis is parallel to the vector of pre-impact velocity of Vehicle $B$.
The local coordinate system $O_{A} x_{A} y_{A} z_{A}$ has its origin $O_{A}$ situated at the centre of mass of Vehicle A and the $O_{A} x_{A}$ axis is parallel to the longitudinal vehicle symmetry plane. The local coordinate system $O_{B} x_{B} y_{B} z_{B}$ is fixed to the body of Vehicle B. The origin $O_{B}$ of this system is situated at the centre of mass of Vehicle B and the $O_{B} x_{B}$ axis is parallel to the longitudinal vehicle symmetry plane. Before the collision, the axes of the local coordinate system $O_{A} x_{A} y_{A} z_{A}$ are parallel to the corresponding axes of the global coordinate system and the local coordinate system $O_{B} x_{B} y_{B} z_{B}$ is rotated in relation to the global system by an angle of $90^{\circ}$ around the vertical axis. 
The quantities whose values were recorded during the crash tests were components of the vectors of acceleration of the centre of vehicle mass $\left(a_{x B}, a_{y B}, a_{z B}\right)$ and of the angular velocity $\left(P_{B}, Q_{B}, R_{B}\right)$ of the vehicle body in relation to the local coordinate system fixed to Vehicle $B$. Angles $\Phi_{B}, \Theta_{B}$ and $\Psi_{B}$ were employed at the transformation of measurement results from system $O_{B} x_{B} y_{B} z_{B}$ to system $O_{G} X_{G} Y_{G} Z_{G}$.

The following relations exist between components of the vector of acceleration of the centre of vehicle mass and components of the vectors of angular velocity of the body of Vehicle B defined in the global and local reference frames:

$$
\left\{\begin{array}{l}
\ddot{X}_{G B} \\
\ddot{Y}_{G B} \\
\ddot{Z}_{G B}
\end{array}\right\}=\left[\begin{array}{lll}
t r_{11} & t r_{12} & t r_{13} \\
t r_{21} & t r_{22} & t r_{23} \\
t r_{31} & t r_{32} & t r_{33}
\end{array}\right] \cdot\left\{\begin{array}{c}
a_{x B} \\
a_{y B} \\
a_{z B}
\end{array}\right\},
$$

where:

$$
\begin{aligned}
& t r_{11}=\cos \left(\Psi_{B}+\frac{\pi}{2}\right) \cdot \cos \Theta_{B} ; \\
& \operatorname{tr}_{12}=\cos \left(\Psi_{B}+\frac{\pi}{2}\right) \cdot \sin \Theta_{B} \cdot \sin \Phi_{B}- \\
& \sin \left(\Psi_{B}+\frac{\pi}{2}\right) \cdot \cos \Phi_{B} ; \\
& \operatorname{tr}_{13}=\cos \left(\Psi_{B}+\frac{\pi}{2}\right) \cdot \sin \Theta_{B} \cdot \cos \Phi_{B}+ \\
& \sin \left(\Psi_{B}+\frac{\pi}{2}\right) \cdot \sin \Phi_{B} ; \\
& t r_{21}=\sin \left(\Psi_{B}+\frac{\pi}{2}\right) \cdot \cos \Theta_{B} ; \\
& t r_{22}=\sin \left(\Psi_{B}+\frac{\pi}{2}\right) \cdot \sin \Theta_{B} \cdot \sin \Phi_{B}+ \\
& \cos \left(\Psi_{B}+\frac{\pi}{2}\right) \cdot \cos \Phi_{B} ; \\
& \operatorname{tr}_{23}=\sin \left(\Psi_{B}+\frac{\pi}{2}\right) \cdot \sin \Theta_{B} \cdot \cos \Phi_{B}- \\
& \cos \left(\Psi_{B}+\frac{\pi}{2}\right) \cdot \sin \Phi_{B} ; \\
& t r_{31}=-\sin \Theta_{B} ; \\
& \operatorname{tr}_{32}=\cos \Theta_{B} \cdot \sin \Phi_{B} ; \\
& \operatorname{tr}_{33}=\cos \Theta_{B} \cdot \cos \Phi_{B} . \\
& \left\{\begin{array}{c}
\dot{\Phi}_{B} \\
\dot{\Theta}_{B} \\
\dot{\Psi}_{B}
\end{array}\right\}=\left[\begin{array}{ccc}
1 & \sin \Phi_{B} \cdot \operatorname{tg} \Theta_{B} & \cos \Phi_{B} \cdot \operatorname{tg} \Theta_{B} \\
0 & \cos \Phi_{B} & -\sin \Phi_{B} \\
0 & \frac{\sin \Phi_{B}}{\cos \Theta_{B}} & \frac{\cos \Phi_{B}}{\cos \Theta_{B}}
\end{array}\right] \cdot\left\{\begin{array}{l}
P_{B} \\
Q_{B} \\
R_{B}
\end{array}\right\} .
\end{aligned}
$$

During the impact, i.e. in the phase of contact between the vehicles, and immediately after this phase, the Vehicle B body solid performs complex motion with a trans- lational velocity $\left\{V_{O B}\right\}$ and an angular velocity of $\left\{\Omega_{B}\right\}$. A preliminary analysis of this motion makes it possible to isolate individual processes, which can be described at the macro- and micro-level. The microscale processes are related to vibrations and wave motions, which are not covered by the scope of this study. During the macro-level analysis of the processes observed, the signals recorded were subjected to low-pass filtration. The filter cut-off frequency is selected to find a compromise between the highest possible accuracy of calculations and the possibility of interpreting the calculation results and of drawing conclusions that would be important and useful for the modelling and reconstruction of road accidents of this kind.

An example of filtration of signals representing the accelerations of the centre of vehicle mass and the angular velocity of the vehicle body, recorded during one of the crash tests carried out $\left(L_{A B}=0.17 \mathrm{~m}\right)$, has been presented in Figure 3. The graphs show results of low-pass filtration at cut-off frequencies of 100, 50 and $25 \mathrm{~Hz}$. In this work, measurement results filtered with $50 \mathrm{~Hz}$ cut-off frequency were taken for analyses.

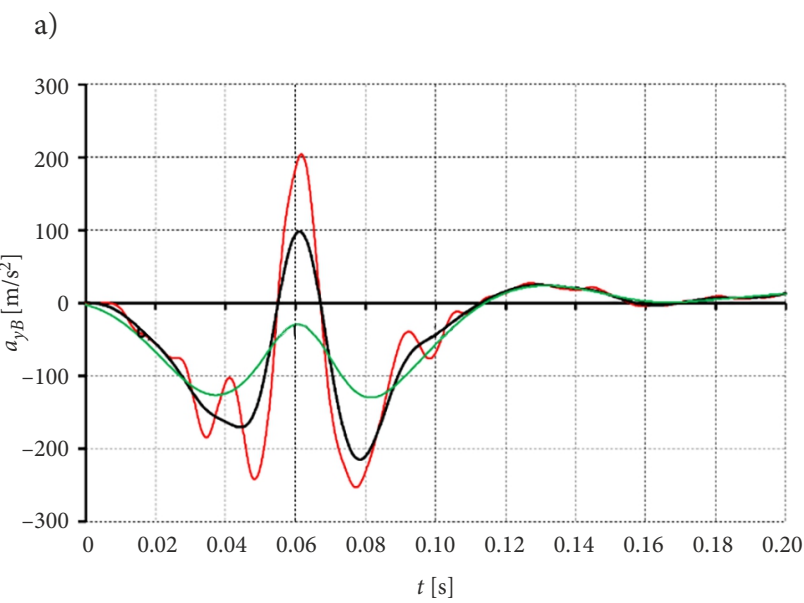

b)

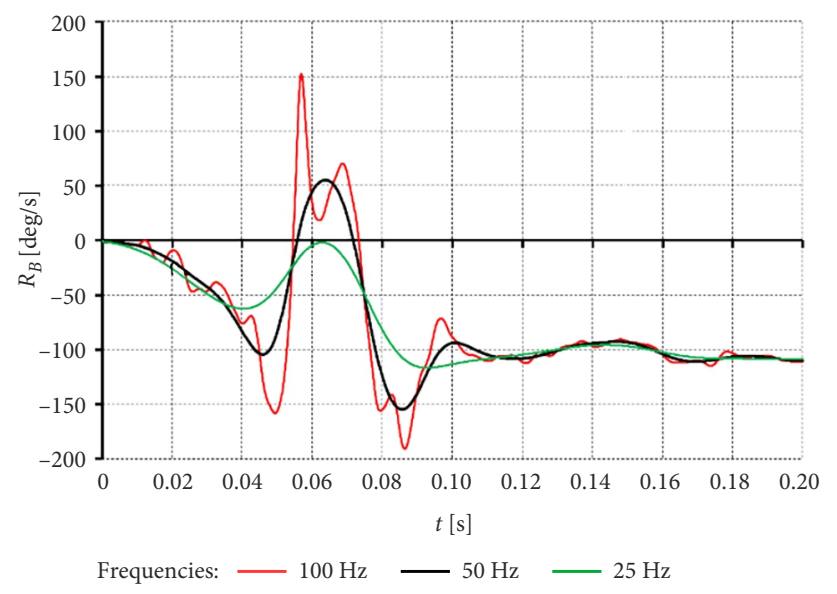

Figure 3. Components of the vectors of acceleration $a_{y B}$ (a) and angular velocity $R_{B}$ (b) of Vehicle $\mathrm{B}$ in the local coordinate system; an example of measurement results obtained from the crash test with $L_{A B}=0.17 \mathrm{~m}$ 


\section{Motion of Vehicle B in the period immediately following the impact}

Figure 4 shows curves obtained from experimental tests and from calculations after transformation of the measurement results to the global coordinate system. The curves represent time histories of velocity $V_{w}$ (Figure $4 \mathrm{a}$ ),

$$
\text { a) }
$$

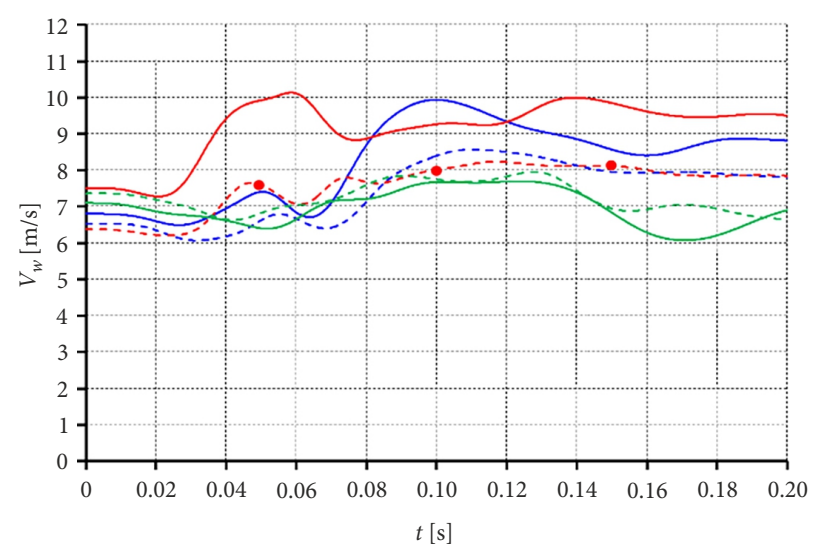

b)

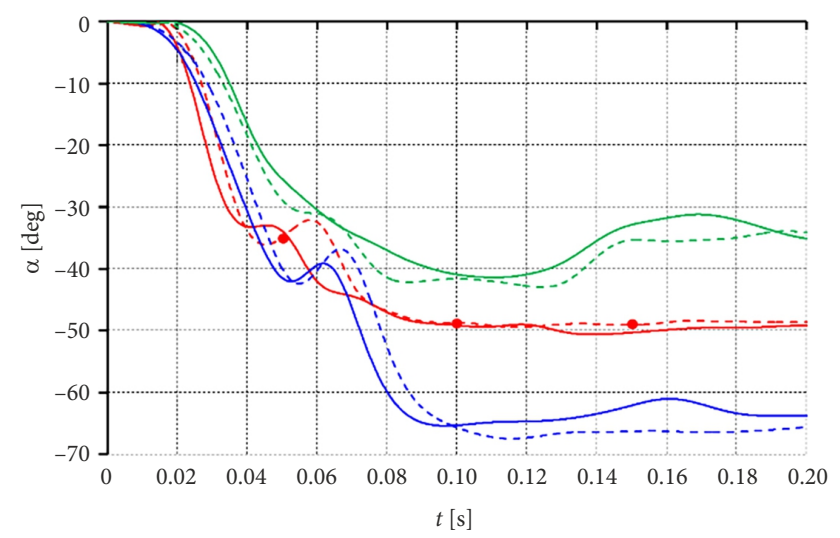

c)

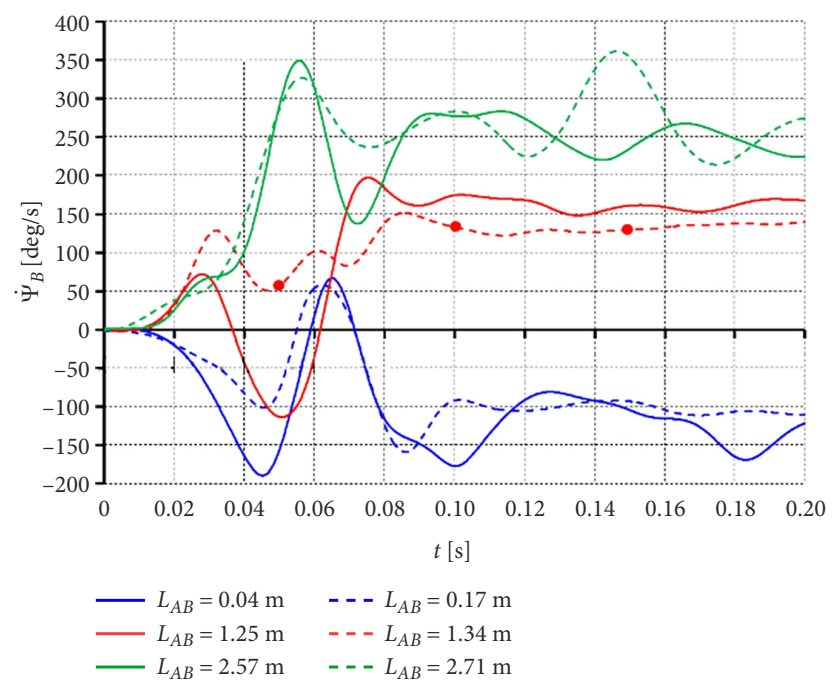

Figure 4. Velocity $V_{w}$ (a), angle of deviation $\alpha$ (b) of the vector of this velocity from the direction of pre-impact vehicle's motion and angular velocity $\dot{\Psi}_{B}$ (c) of the body of Vehicle B angle $\alpha$ (Figure $4 \mathrm{~b}$ ), and angular velocity $\dot{\Psi}_{B}$ (Figure $4 \mathrm{c}$ ) for the six crash tests. These curves make it possible to determine values of the parameters that describe the motion of Vehicle B in the road surface plane at every instant within the period $0 \ldots 0.2 \mathrm{~s}$, i.e. in the initial phase of the collision process.

Individual components of the vector of velocity $\dot{X}_{G B}$, $\dot{Y}_{G B}$ were determined from Equation (1). Velocity $V_{w}$ and angle $\alpha$ were determined from equations:

$$
\begin{aligned}
& V_{w}=\sqrt{\dot{X}_{G B}^{2}+\dot{Y}_{G B}^{2}} ; \\
& \alpha=\operatorname{arctg}\left(\frac{\dot{Y}_{G B}}{\dot{X}_{G B}}\right) .
\end{aligned}
$$

Based on results of these calculations, their practical illustration for a few specific instants has been presented in Figure 5. A few successive positions of Vehicle B during the crash test have been shown for $L_{A B}=1.34 \mathrm{~m}$, with marking the components $\dot{X}_{G B}, \dot{Y}_{G B}$ of velocity vector $V_{w}$ and angles $\alpha$ and $\Psi_{B}$. The calculation results presented have been marked with dots on the curves plotted in Figure 4.

The curves shown in Figure 6 make it possible to identify changes in the position of Vehicle B in the initial phase of the collision process, i.e. changes from the state for $t=0 \mathrm{~s}$. These positions are defined by three parameters: $X_{G B}$ (Figure 6a), $Y_{G B}$ (Figure 6b) and $\Psi_{B}$ (Figure 6c), i.e. the lateral and longitudinal displacements of the centre of vehicle mass and the angle of deviation of the vehicle body from the $O_{G} Y_{G}$ axis in result of yaw (rotation of the vehicle body around an axis parallel to the $O_{G} Z_{G}$ axis).

Figure $6 \mathrm{~d}$ is an illustration for the general desription of the motion of Vehicle B during the period $0 \ldots 0.2 \mathrm{~s}$. It shows the trajectories of the centre of vehicle mass; in combination with Figure $6 \mathrm{c}$ (angle $\Psi_{B}$ ), it provides complete information about the vehicle position in the global coordinate system. The graph shows that the displacement of the centre of mass of Vehicle B in the time interval under analysis did not exceed $1.4 \mathrm{~m}$. After this period, Vehicle B moves freely and the initial values of the parameters that characterize the vehicle motion in this phase are determined by the calculation results, which will be presented hereafter. The said calculation results are sufficient to define the post-impact vehicle motion in the road surface plane $O_{G} X_{G} Y_{G}$. The data collected and the calculation method adopted make it also possible to determine the vehicle motion in the plane perpendicular to the road surface; however, the vehicle motion in this plane has rather a small impact on the post-impact vehicle trajectory and it was not considered at this stage of the work.

\section{Approximation of the experiment and calculation results}

The experimental test results, after transformation to the global coordinate system, have been presented in Figures 4 and 6 . The curves shown in the graphs were treated 
a)

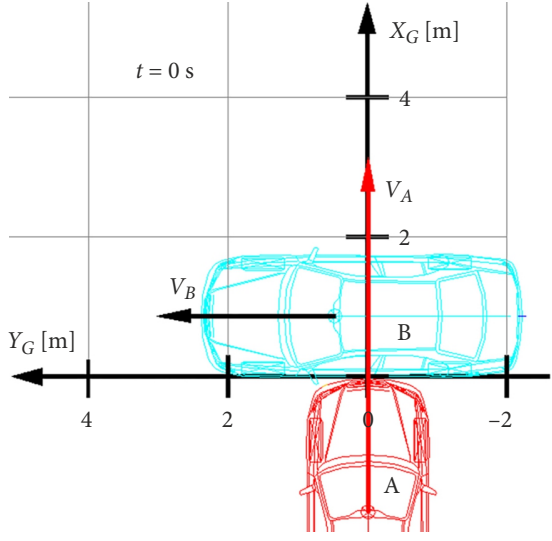

c)

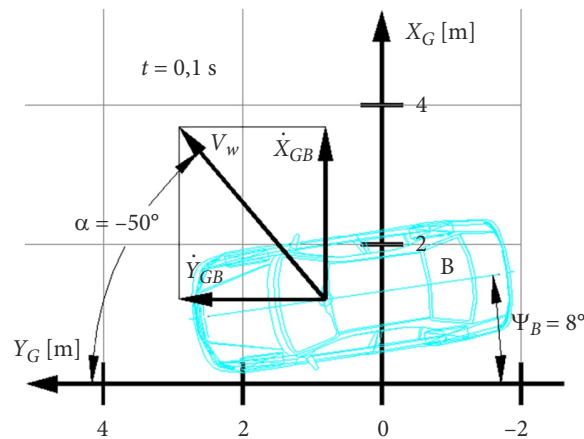

b)

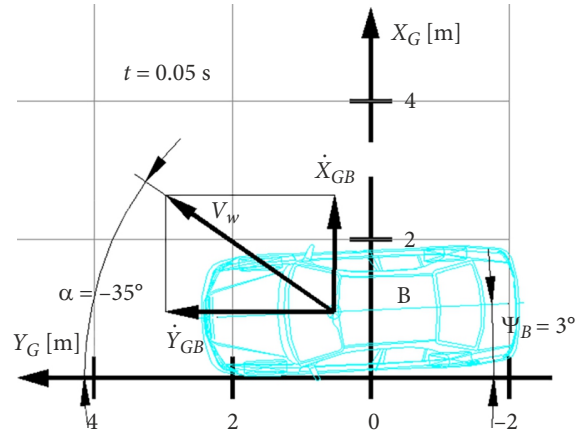

d)

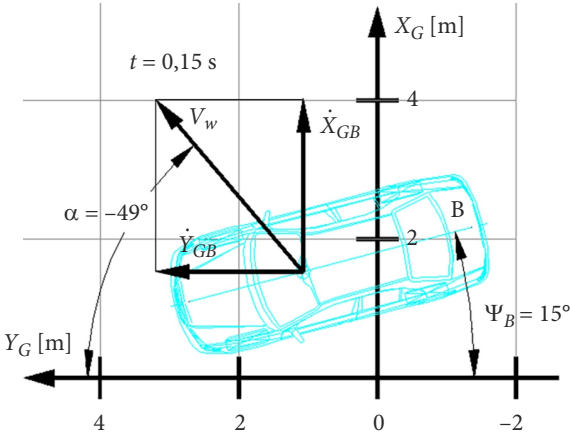

Figure 5. Example graphical illustration of results of calculations (based on data taken from Figure 4), i.e. the positions of Vehicle B and of the velocity vector $V_{w}$ and its components in the global coordinate system, for a few selected instants; data taken from the test where $L_{A B}=1.34 \mathrm{~m}$

a)

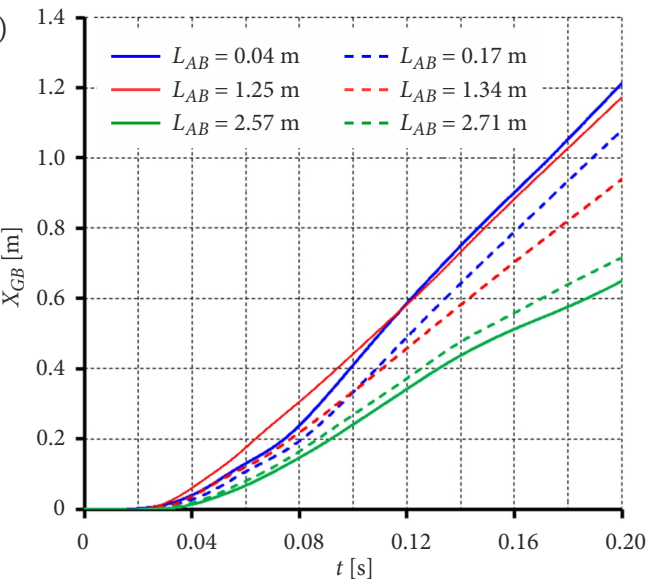

c)

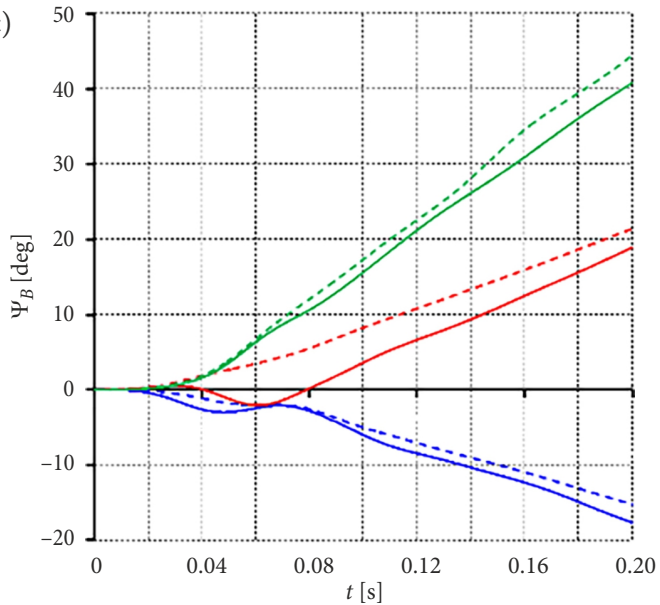

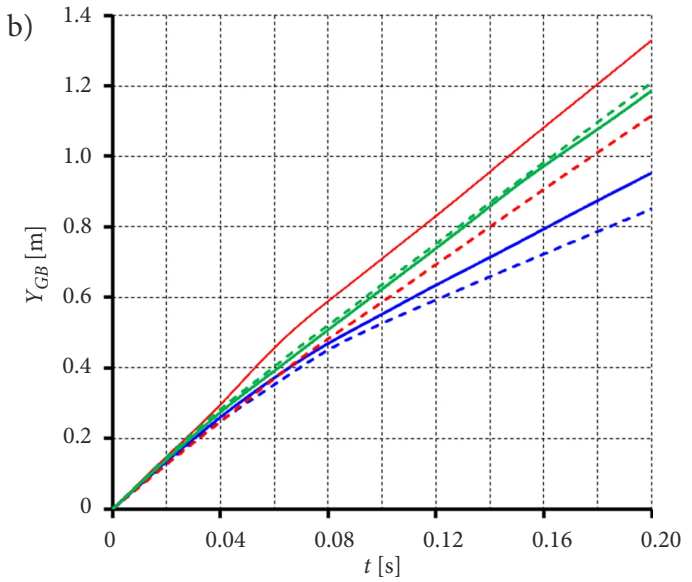

d)

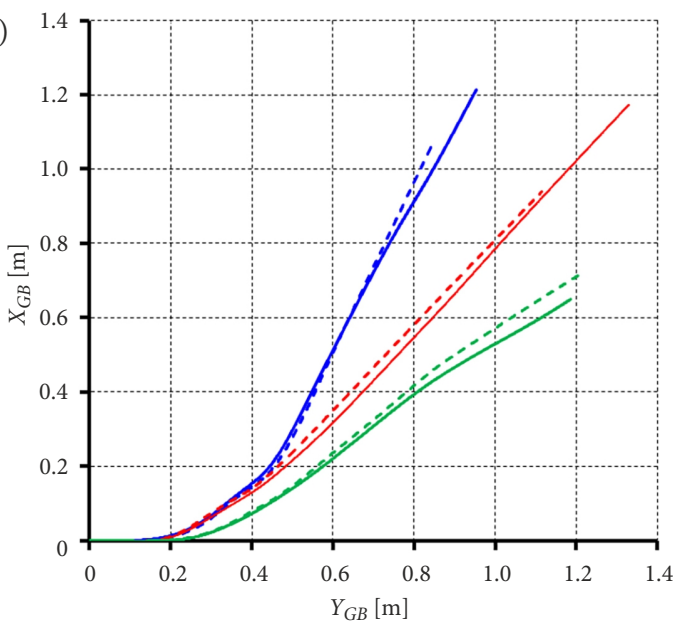

Figure 6. Lateral and longitudinal displacement $X_{G B}(\mathrm{a})$ and $Y_{G B}(\mathrm{~b})$, respectively of the centre of mass of Vehicle B, angle of yaw $\Psi_{B}$ (c) of the body of Vehicle B, and trajectory of the centre of mass of Vehicle B (d) in the global coordinate system 
as functions of time $W(t)$ and then utilized to calculate the average value $W\left(T_{k}\right)$. Before this operation, the values of functions $W(t)$ were normalized so that all the results could be treated as obtained from tests carried out at an identical vehicle velocity value. Thus, the average value of velocity $\left\{V_{A}\right\}$ (Table 1 ) was calculated and then the normalization was done as follows:

$$
W(t) \rightarrow W(t) \cdot \frac{\bar{V}_{A}}{V_{A}} .
$$

When the average value $W\left(T_{k}\right)$ was calculated for the $W(t)$ curve after normalization, several characteristic time intervals were selected, as shown in Figure 7 and in Table 2.

The $W\left(T_{k}\right)$ value is the average value of function $W(t)$ in time interval $t_{i} \ldots t_{j}$. In result of calculations, a set of $n \times k$ average values $W_{n k}$ was obtained for each of the $W(t)$ functions, where:

$-n=1, \ldots, 6$ (6 crash tests, for individual $L_{A B}$ values given in Table 1 in succession);

$-n=1, \ldots, 4$ (4 time intervals for averaging the $W(t)$ functions, as specified in Table 2.

The relation between values $W_{n k}$ and $L_{A B}$, i.e. the point of impact against the vehicle side, was determined with the use of the regression method. The regression function was modelled by a second degree polynomial and the coefficients of the polynomial were determined by the least squares method. For the set of the $W_{n k}$ values, four regression function curves were determined for each of the $W(t)$ functions, for $k=1,2,3,4$ in succession. The curves have been shown in Figure 8. The quality of the regression function models was assessed on the grounds of the values of the coefficient of determination $R^{2}$; the average values of this coefficient, calculated for 4 regression curves, have been given in individual graphs in Figure 8 .

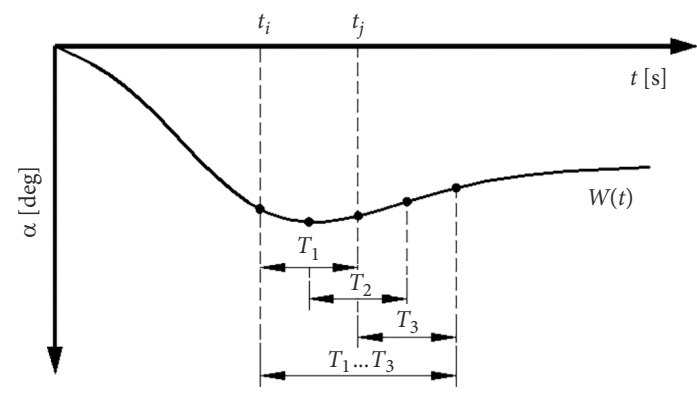

Figure 7. Example function $W(t)$ with time intervals having been marked

Table 2. Time intervals for the calculation of averaged values of functions $W(t)$

\begin{tabular}{|l|c|c|c|c|}
\hline Description & $T_{1}$ & $T_{2}$ & $T_{3}$ & $T_{1} \ldots T_{3}$ \\
\hline $\begin{array}{l}\text { Time } \\
\text { interval } \\
t_{i} \ldots t_{j}[\mathrm{~s}]\end{array}$ & $0.08 \ldots 0.12$ & $0.10 \ldots 0.14$ & $0.12 \ldots 0.16$ & $0.08 \ldots 0.16$ \\
\hline
\end{tabular}

\section{Recapitulation and conclusions}

Usually, the following information is available that can be used for the modelling and reconstruction sense of the post-impact motion of a motor vehicle struck on its side at a road intersection (Prochowski et al. 2014; Wach 2014):

- post-accident position of the vehicle;

- location of the vehicle collision;

- location of the point where the vehicle side was struck by another vehicle (point of impact against the vehicle side), i.e. location of the vehicle side deformation area $\left(L_{A B}\right)$;

- data that facilitate the vehicle identification and thus enable determining the mass parameters and important dimensions of the vehicle.

According to the experimental tests and calculations carried out, the values and profiles of the following functions characterizing the post impact vehicle motion have been obtained for passenger cars of a certain category (i.e. whose unladen mass, overall length, and body shape are similar to those of the vehicles under tests):

- position of the impacted vehicle at the end of the phase of contact with the impacting vehicle;

- energy of the translational and rotational motion of the impacted vehicle at the beginning of the free motion phase, if the vehicle mass parameters are known;

- value, direction, and sense of the vector of resultant velocity of the centre of mass of the impacted vehicle and of the vector of angular velocity of rotation of the impacted vehicle around its vertical axis.

The curves presented in Figure 8 show, in a graphical form, the relation between the location of the point of impact against the vehicle side and the parameters of motion of the impacted vehicle.

In the current road accident reconstruction procedures, results of single crash tests are usually taken into account and they are often arbitrarily applied to individual specific accidents under analysis. In contrast, the results obtained from a big series of crash tests and from a regression analysis carried out provide a possibility of determining (within the reconstruction of road traffic incidents) the values of parameters of the state of motion of the impacted vehicle for any location of the point of impact against the vehicle side. Thanks to this, a possibility of considerable reduction in the uncertainty of simulation calculations carried out within the reconstruction of road accidents has been created.

The identified relation between the location of the point of impact against the vehicle side and the parameters of motion of the impacted vehicle can also be used for the creation of new models for the optimization of the simulation programs employed at the reconstruction of road traffic incidents. 
a)

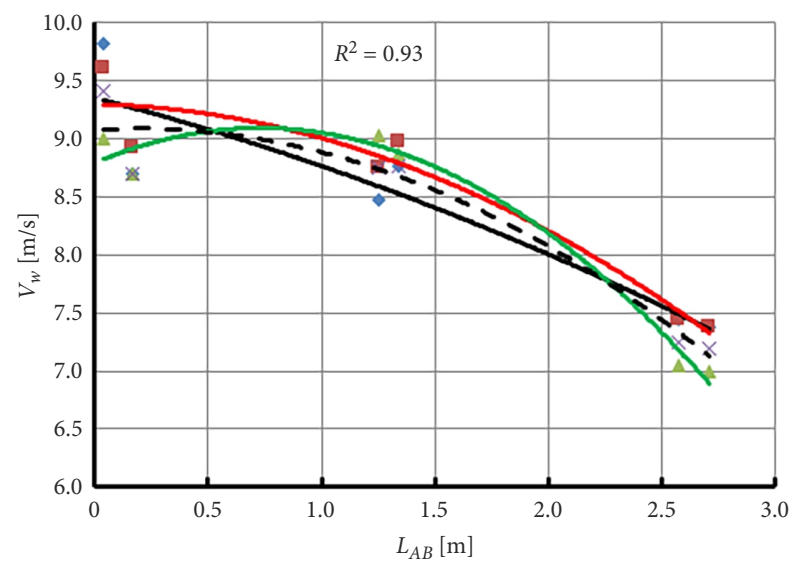

c)

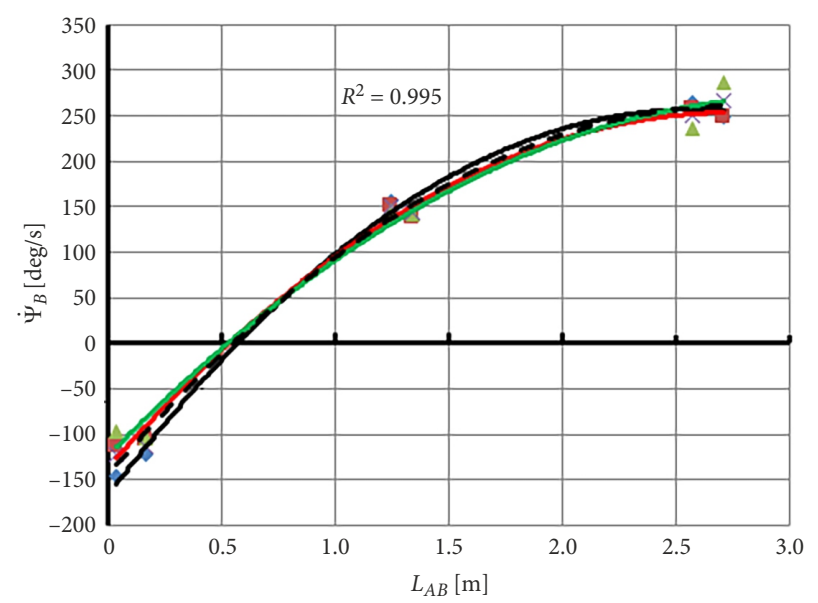

e)

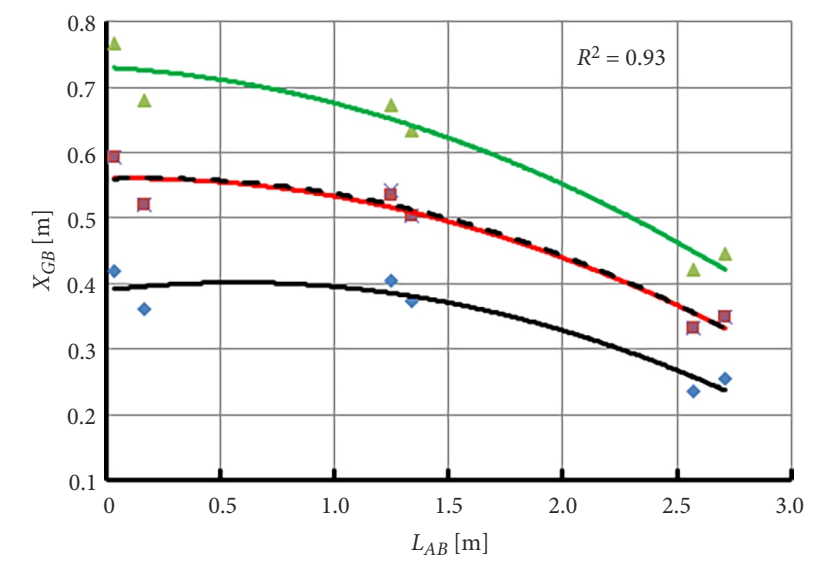

b)

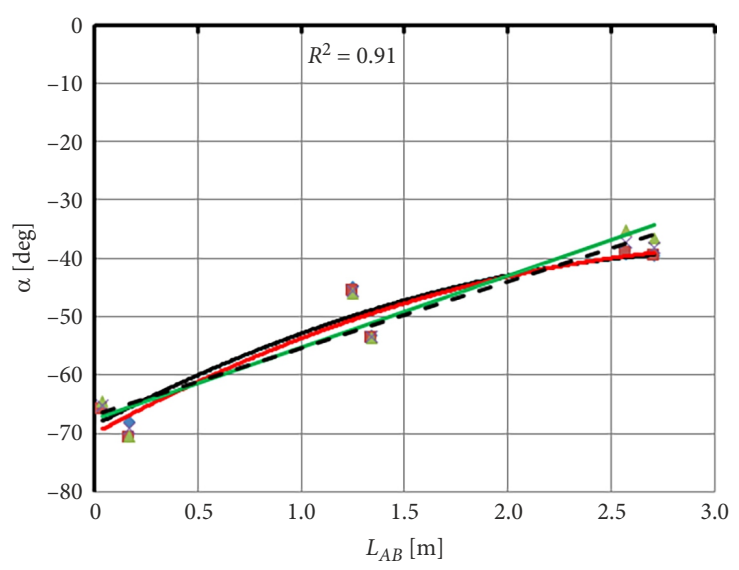

d)

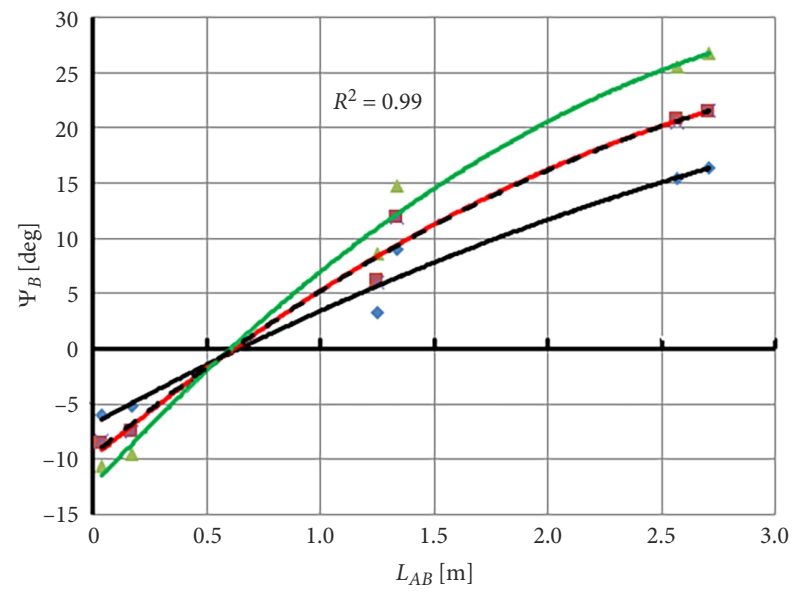

f)

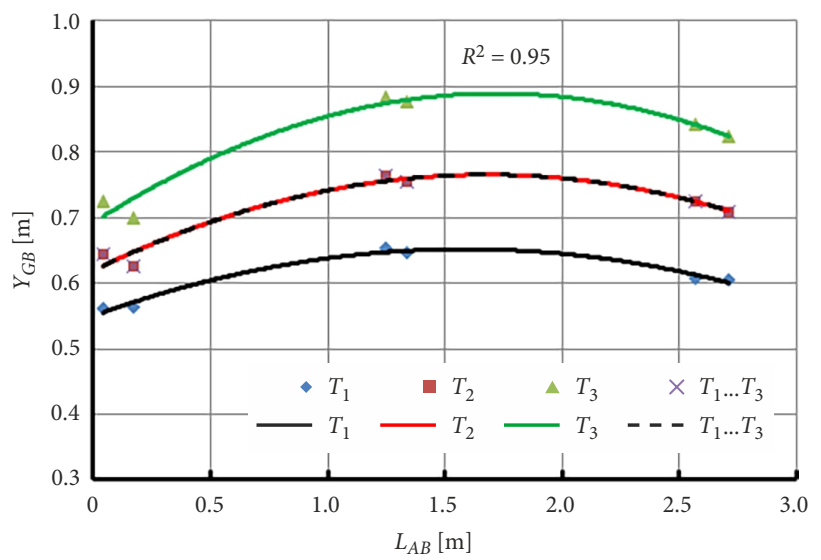

Figure 8. Regression curves depending on the point of impact against the vehicle side $\left(L_{A B}\right)$ : a - velocity $V_{w}$; b angle $\alpha$; - angular velocity $\dot{\Psi}_{B} ; \mathrm{d}$ - yaw angle $\Psi_{B}$; e - lateral displacement $X_{G B} ; \mathrm{f}$ - longitudinal displacement $Y_{G B}$

\section{References}

Bailey, M.; Lawrence, J.; Fowler, S.; Williamson, P.; Cliff, W.; Nickel, J. 2000. Data from five staged car to car collisions and comparison with simulations, SAE Technical Paper 2000-010849. https://doi.org/10.4271/2000-01-0849

Brach, R. M.; Brach, R. 2011. Vehicle Accident Analysis and Reconstruction Methods. SAE International. $442 \mathrm{p}$.

Brach, R. M.; Welsh, K.; Brach, R. 2007. Residual crush energy partitioning, normal and tangential energy losses, SAE Technical Paper 2007-01-0737.

https://doi.org/10.4271/2007-01-0737

Cliff, W. E.; Moser, A. 2001. Reconstruction of twenty staged collisions with PC-CRASH's optimizer, SAE Technical Paper 2001-01-0507. https://doi.org/10.4271/2001-01-0507

Gidlewski, M.; Prochowski, L.; Zielonka, K. 2015. Analysis of the influence of motor cars' relative positions during a right angle crash on the dynamic loads acting on car occupants and 
the resulting injuries, in 24th International Technical Conference on the Enhanced Safety of Vehicles (ESV), 8-11 June 2015, Gothenburg, Sweden, 1-12. Available from Internet:

https://www-esv.nhtsa.dot.gov/Proceedings/24/isv7/main.htm

Johnson, N.; Gabler, H. C. 2011. Evaluation of WinSMASH accuracy in NHTSA side crash test reconstructions, in 22nd International Technical Conference on the Enhanced Safety of Vehicles (ESV), 13-16 June 2011, Washington, DC, US, 1-8. Available from Internet:

https://www-esv.nhtsa.dot.gov/Proceedings/22/isv7/main.htm

McHenry, B.; McHenry, R. 1997. CRASH-97 - refinement of the trajectory solution procedure, SAE Technical Paper 970949. https://doi.org/10.4271/970949

Prochowski, L.; Unarski, J.; Wach, W.; Wicher, J. 2014. Podstawy rekonstrukcji wypadków drogowych. Warszawa: Wydawnictwa Komunikacji i Łączności. 360 s. (in Polish).

Seidl, M.; Tomek, M. 2015. Traffic accidents rate in the Slovak Republic in 19th International Scientific Conference on Transport Means: Proceedings of the International Conference, 22-23 October 2015, Kaunas, Lithuania, 257-260.

Wach, W. 2014. Wiarygodność strukturalna rekonstrukcji wypadków drogowych. Kraków: Wydawnictwo Instytutu Ekspertyz Sądowych. 194 s. (in Polish). 